\title{
Xanthogranulomatous cystitis associated with inflammatory bowel disease
}

\author{
Doreen E. Chung, MD, FRCSC,; Lesley K. Carr, MD, FRCSC;; Linda Sugar, MD, FRCPC; ; \\ Michelle Hladunewich, MD, FRCPC, MSc, BSc;: Leslie A. Deane, MBBS, FRCSC ${ }^{\varsigma}$
}

\begin{abstract}
Xanthogranulomatous inflammation is a benign condition characterized by the presence of multinucleated giant cells, chronic inflammatory cells and lipid-laden macrophages, known as xanthoma cells. Only 22 cases of xanthogranulomatous cystitis (XGC) have been reported in the Japanese and English literature. In this report, we describe the twenty-third case of XGC and the third case associated with inflammatory bowel disease (IBD). A 50-year-old woman with quiescent Crohn's disease was incidentally found to have a bladder mass on ultrasound. The lesion was resected through a transurethral approach. Pathology demonstrated XGC. At 3 months post-resection, there was no evidence of recurrence adjacent to the previous resection scar.
\end{abstract}

Can Urol Assoc J 2010;4(4):E91-93

\section{Introduction}

Xanthogranulomatous inflammation is a benign condition characterized by the presence of multinucleated giant cells, chronic inflammatory cells and lipid-laden macrophages known as xanthoma cells. ${ }^{1}$ Xanthogranulomatous pyelonephritis is not uncommon. However, only 22 cases of xanthogranulomatous cystitis (XGC) have been reported in the Japanese and English literature. In this report, we describe the twenty-third case of XGC and the third case associated with inflammatory bowel disease (IBD).

\section{Case report}

A 50-year old woman with Crohn's disease had an abdominal and pelvic ultrasound study performed for right-sided abdominal pain in 2004 (Fig. 1). The pain had been present for months, was moderate in severity and was difficult to localize. She denied having had any lower urinary tract symptoms, hematuria or previous urinary tract infections. Papillary projections from the dome of the bladder were seen on the study. In retrospect, this mass had been present on contrast computed tomography imaging from 2002, which was performed to further evaluate an incidental left renal cyst seen on ultrasound. However, it was not commented on in the original study report. The patient's past medical history included Crohn's disease diagnosed 4 years prior, recurrent pancreatitis, pancreas divisum, chronic renal failure secondary to interstitial disease, and papillary necrosis. She had not had any prior abdominal surgery for IBD.

On physical examination, no mass was palpable. Cystoscopy was performed to further evaluate the bladder lesions. Extensive papillary lesions were observed covering most of the dome and anterior wall of the bladder, some with calcified tips (Fig. 3). Urine culture was negative for bacteria as well as for acid-fast bacilli. Urine cytology at the time of cystoscopy demonstrated acute inflammatory cells and histiocytes. Serum antinuclear antibodies, compliment levels, eosinophils and hepatitis serology were all within normal limits.

Complete endoscopic resection of the lesions was done. Muscle was included in the pathology specimen. Postoperative course was unremarkable.

Histological examination of the specimen was consistent with XGC. The lamina propria was expanded by sheets of foamy histiocytes admixed with some lymphocytes and occasional eosinophils. The histiocytes, stained positive for CD68, confirmed the presence of foamy histiocytes, and there were no Michaelis-Gutmann bodies identified. Michaelis-Gutmann bodies are present in malakoplakia, but not in XGC. There was no evidence of malignancy.

At 3 months post-resection, there was no evidence of recurrence adjacent to the previous resection scar (Fig. 4). The patient continues to be asymptomatic and has declined both repeat transurethral resection of a bladder tumour and partial cystectomy. Although optimal treatment of choice is unknown for this condition, curative treatment of choice is surgery, usually partial cystectomy. ${ }^{2}$

The patient recently returned for follow-up 5 years after the original resection. Negligible urinary tract symptoms were reported and the patient was not having abdominal pain. During cystoscopy, the previous transurethral resection site demonstrated only scar, without any sign of progression. 


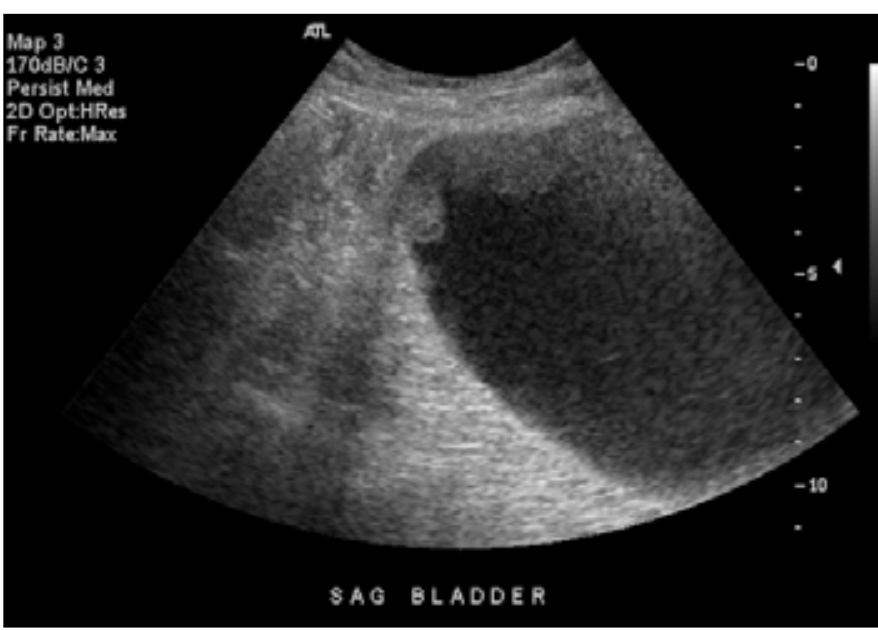

Fig. 1. 2004 Sagittal view of the bladder. Note the papillary lesions at the dome and anterior wall of the bladder.

\section{Discussion}

Xanthogranulomatous cystitis is a rare inflammatory disorder. Multiple theories about its etiology have been proposed, including chronic irritation of the urachal remnant, chronic bacterial infection, immunological disorders, and abnormal lipid metabolism. ${ }^{2}$ Most cases are associated with an urachal remnant and involve the bladder dome. ${ }^{2}$ This condition has also been reported to be associated with adjacent malignancy from the gastrointestinal or genitourinary tract. ${ }^{3}$

Strikingly, our case is the third to occur in a woman with IBD. ${ }^{2,4}$ All 3 patients had disease of the bladder dome and none had an urachal remnant. Since partial cystectomy was not done, it is not definitively known whether this particular patient had an urachal remnant. The mean age of the other 2 patients with IBD and XGC was 50; both of these cases were treated with partial cystectomy and in 1 case the patient remained disease-free for more than 15 years. ${ }^{2}$ The fact that our patient had radiographic evidence of the lesion 2 years prior to resection further emphasizes the benign nature of the disease.

The association between IBD and xanthogranulomatous cystitis supports the theory that XGC is a consequence of chronic inflammation and altered regulation of the immune response. It may be that chronic inflammation of the gut leads to XGC through diffusion of active inflammatory cytokines in adjacent bowel.

\section{Conclusion}

This case of XGC associated with IBD supports the theory that XGC may be a result of an immunological disorder. The patient's lack of recurrence following local resection suggests that XGC is benign.

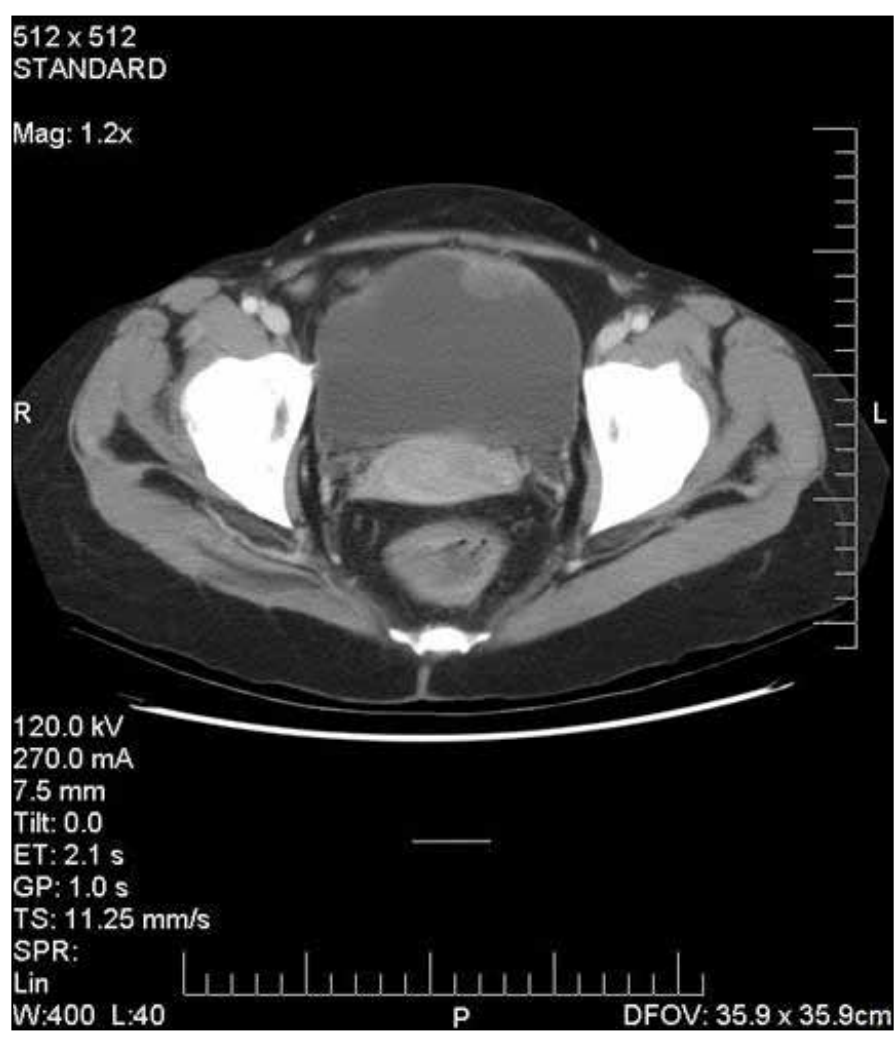

Fig. 2. 2002 Computed tomography image. Note the mass arising from the anterior wall of the bladder.

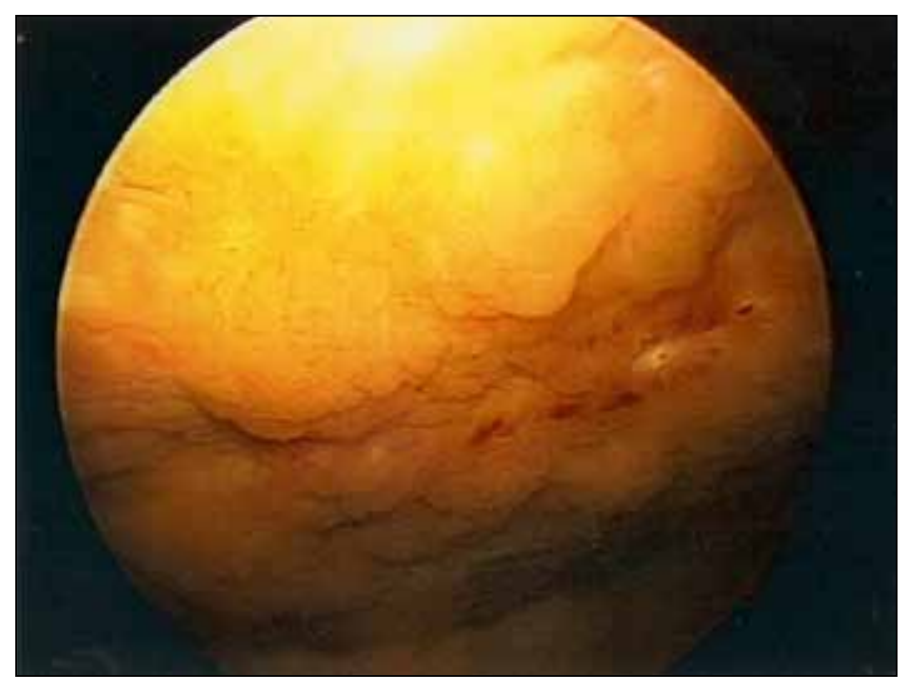

Fig. 3. Cystoscopy image of xanthogranulomatous cystitis, with the appearance of papillary projections, on the dome of the bladder.

Clinical Assistant Professor, Section of Urology, The University of Chicago, Chicago, IL; ${ }^{\dagger}$ Assistant Professor, University of Toronto, Department of Surgery, Division of Urology, Sunnybrook Health Sciences Centre, Toronto, ON; ₹Staff Pathologist, Sunnybrook Health Sciences Centre, Professor, Department of Lab Medicine and Pathobiology, University of Toronto, Toronto, ON; ${ }^{2}$ Assistant Professor of Medicine, University of Toronto, Divisions of Critical Care \& Nephrology, Acute Dialysis Director, Sunnybrook Health Sciences Centre, Toronto, ON; §Assistant Professor of Urology, Director of Laparoscopy, Endourology and Robotic Surgery, Department of Urology, University of Illinois at Chicago, Chicago IL 


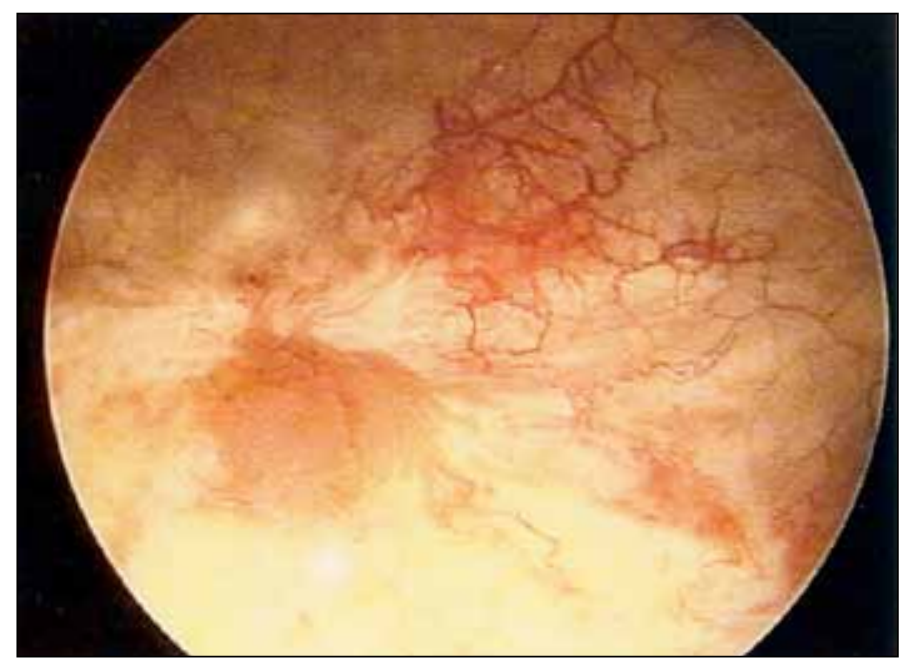

\section{References}

1. Oberling C. Retroperitoneal xanthogranuloma. Am J Cancer 1935;23:477-89.

2. Tan L, Chiang $C$, Huang $G$, et al. Xanthogranulomatous cystitis: a case report and review of the literature. Int Urol Nephrol 1994;26:413-7.

3. Garcia A, Florentine B, Simons A, et al. Xanthogranulomatous cystitis as a cause of elevated carcinoembryonic antigen mimicking recurrent colorectal cancer. Report of a case. Dis Colon Rectum 1996;39:1051-4.

4. Walther M, Glenn J, Vellios F. Xanthogranulomagous cystitis. J Urol 1985;134:745-6.

Correspondence: Dr. Doreen E. Chung, Clinical Assistant Professor, Section of Urology, The University of Chicago, 1500 S. California, Department of Surgery - F944, Chicago, II 60608; fax: 773-2576548; doreen_chung@yahoo.ca

Fig. 4. Cystoscopy image 3 months post-resection.

Competing interests: None declared.

This paper has been peer-reviewed. 